\title{
Overcoming the organ donation dilemma
}

Jason T. Siegel and Eusebio M. Alvaro

Understanding organ donation: Applied behavioral science perspectives.

Wiley-Blackwell, 2010, 392 pp.

US\$124.95 (hbk); ISBN 978-1-4051-9213-2

The lack of correspondence between attitudes and behavior has received much attention in psychology research, and it is a problem most vividly demonstrated in the field of organ donation. Surveys conducted by the Gallup Organization consistently show that over $90 \%$ of people support the concept of organ donation yet, in practice, consent rates for organ donation do not reflect this support. In the book, Understanding organ donation, Siegel and Alvaro have drawn together an impressive collection of chapters detailing research projects that have attempted to solve this critical attitude-behavior problem. As its' title suggests this edited volume focuses on the application of psychological knowledge to understand and increase organ donation. It serves as an excellent introduction to an emerging field in health psychology for interested researchers and practitioners alike. One particularly noteworthy chapter in this regard is Ganikos' detailed and informative overview of the field which effectively sets the scene for the remaining chapters. However, this book is much more than a user-friendly introduction to understanding organ donation.

Aside from the introduction and overview chapters, the book is comprised of three additional sections encompassing media and community interventions (Part I), organizational interventions (Part II), and broad perspectives and future directions (Part III). The two sections on organ donation interventions offer a wealth of knowledge about the expected and unexpected challenges and issues faced when conducting applied research. Alvaro and Siegel begin Part I with a useful review of organ donation mass media campaigns, drawing together underlying theory and details of campaigns since the 1980's with a focus on recent campaigns for Hispanic Americans as an exemplar. The cultural perspective is well represented in the subsequent intervention chapters outlining media and/or community campaigns with African American, Alaska Native, American Indian, First Nation, and Chinese American communities. I particularly enjoyed reading Allen and 
Stillwater's chapter on creating a public health awareness program for the Alaska Native community and the importance of using messages to promote organ donation and transplantation that are consistent with deeply ingrained cultural values - in this case the tradition of giving and sharing.

Part II continues with a presentation of organizational interventions in a range of settings involving parties directly and indirectly associated with the donation/transplantation process (e.g., Paykin, Politoski, and Singleton-Driscoll's chapter on improving communication between recovery professionals and funeral directors) and with potential donors (e.g., Feeley, Anker, Vincent, and Williams' chapter detailing an intervention with college students). Morgan's chapter concludes the intervention sections with an invaluable discussion of the challenges of conducting and evaluating organ donation campaigns, highlighting the importance of maintaining intervention fidelity. Readers will also find Fischer's chapter (Part III) on evaluating the effects of community-based health interventions to be of interest. In reading these intervention chapters, I was struck by the tenacity of all of the researchers in their efforts to create and implement a range of novel interventions. The persistence of Johnson and Webber in pursuing a Donate Life workplace partnership with the Home Care Association of Louisiana to increase participation in the Louisiana donor registry is especially commendable in the face of the aftermath of Hurricane Katrina. Indeed, the outstanding feature of all of these chapters in Parts I and II is that they share valuable guidance and suggestions for overcoming or avoiding the challenges inherent in designing, and especially conducting, an organ donation-related intervention. Many of the challenges described and the suggestions provided to overcome these challenges and develop a successful intervention will resonate with applied researchers generally. This book is, therefore, a worthwhile read for any researcher or practitioner considering developing or implementing a health behavior intervention or campaign.

From an international organ donation research perspective, the downside of these chapters is their primary focus on increasing participation in US donor registries. Given the use of first-person consent registries in the US (aptly described in Downing and Jones's chapter on the first-person consent Ohio donor registry), and the advantages of having an objective 
outcome measure of consent, this focus is certainly understandable. However, this focus does not reflect the shifting trend in the international research literature of talking with family about the donation preference as a way of increasing consent for donation, especially in countries where first-person consent registries may exist but the family decision is still paramount. My disappointment with the lack of interventions designed to increase family discussion was somewhat tempered by Part III of the book (broad perspectives and future directions) which includes a chapter on the value of adopting qualitative research strategies to study family discussion about organ donation. In this excellent chapter, Pomerantz draws on the broader literature about interpersonal discussion of sensitive health topics to inform a qualitative case study with university students about the dynamics of family organ donation discussions. This insightful approach will go some way towards increasing our understanding of the challenges that people face when attempting to communicate their donation wishes to family, and the factors that may prevent a person from initiating a conversation about organ donation in the first place.

Another highly recommended chapter in Part III, authored by Pace Jones and Bosch, gives the practitioner perspective on collaborating with applied researchers to meet the common goal of increasing organ donation rates. This chapter serves as an interesting commentary on improving collaborative relationships with researchers and offers several strategies to promote an effective and successful partnership, making it a worthwhile read for researchers and community organizations considering working together. For those interested in living donation, the chapter by Waterman and Rodrigue will be a welcome addition. This chapter asks and answers a very important question that has plagued living organ donation researchers for some time - how should we ethically and effectively advance the promotion of living donation? In this chapter, the authors propose a socialecological transplant model as a theoretical framework for living donor kidney transplantation (LDKT), outline LDKT education opportunities for health professionals, and provide recommendations for promoting LDKT. Siegel, Alvaro and Hohman, report on their efforts to understand why positive attitudes towards organ donation do not always translate into positive donor registrations. In this thoroughly enjoyable and important chapter, the authors describe their use of the Theory of Planned Behavior model to understand the attitude-behavior discrepancy, with a particular focus on the utility of 
perceived behavioral control as a mechanism for behavior change. Based on their experiences, the authors suggest that registration is most likely to occur if four key factors are present ("an immediate and complete registration opportunity, information, focused engagement, and favorable activation", p. 326) - referred to as the IIFF model. This promising model is likely to be an asset to future researchers seeking to maximize their chances of achieving positive behavioral outcomes in behavior change interventions.

Crano's chapter concludes the section with a commentary on the progress of organ donation research to date and highlights the importance of theory in applied research endeavors. I would like to conclude this review by echoing Crano's sentiments about the critical importance of theory development in this domain. As researchers attempting to solve the organ donation dilemma, we have made great strides in understanding and identifying targets for intervention, and this seminal volume of research is no exception; however, a continued focus on theory development should go hand-in-hand with applied research and experimentation to facilitate behavior change to ensure that, ultimately, willingness translates into consent for organ donation.

MELISSA K. HYDE

Queensland University of Technology, Australia 